\title{
ACOUSTIC FLAW TRIANGULATION ON A \\ THIN SPHERICAL SHELL: AN \\ ANALYTICAL SOLUTION
}

H. W. Blake

C. M. Davenport

\section{UNION CARBIDE CORPORATION}

\section{NUCLEAR DIVISION \\ OAK RIDGE Y-12 PLANT}

operated for the ATOMIC ENERGY COMMISSION under U. S. GOVERNMENT Contract W-7405 eng 26

UNION

CARBIDE
OAK RIDGE Y.12 PLANT

P. O. BOX $Y$

OAK RIDGE, TENNESSEE 37830 


\section{DISCLAIMER}

This report was prepared as an account of work sponsored by an agency of the United States Government. Neither the United States Government nor any agency Thereof, nor any of their employees, makes any warranty, express or implied, or assumes any legal liability or responsibility for the accuracy, completeness, or usefulness of any information, apparatus, product, or process disclosed, or represents that its use would not infringe privately owned rights. Reference herein to any specific commercial product, process, or service by trade name, trademark, manufacturer, or otherwise does not necessarily constitute or imply its endorsement, recommendation, or favoring by the United States Government or any agency thereof. The views and opinions of authors expressed herein do not necessarily state or reflect those of the United States Government or any agency thereof. 


\section{DISCLAIMER}

Portions of this document may be illegible in electronic image products. Images are produced from the best available original document. 
This report was prepared as an account of work sponsored by the United States Government. Neither the United States nor the United States Atomic Energy Commission, nor any of their employees, nor any of their contractors, subcontractors, or their employees, makes any warranty, express or implied, or assumes any legal liability or responsibility for the accuracy, completeness or usefulness of any information, apparatus, product or process disclosed, or represents that its use would not infringe privately owned rights. 


\section{ACOUSTIC FLAW TRIANGULATION ON A THIN SPHERICAL SHELL: AN ANALYTICAL SOLUTION}

H. W. Blake

C. M. Davenport

NOTICE 3 account of work This report was prepared stales Covernment. Nelle Thonsored by the Unit the United States Eny of the United States Research and Dees, not any of thes, makes ony theit emplors, or their employecs, makes sny legal subcranty, express or implied, accuracy, completeness Thbility or responsibilly rom the soparatus, product on or usefulness of any in represents that its use wowd not process disclosed, oumed rights.

Oak Ridge Y-12 Plant

P.O. Box Y, Oak Ridge, Tennessee 37830

operated for the U.S. ATOMIC ENERGY COMMISSION

bY UNION CARBIDE CORPORATION-NUCLEAR DIVISION

under Contract W-7405-eng-26.

Date Issued - December 30, 1971
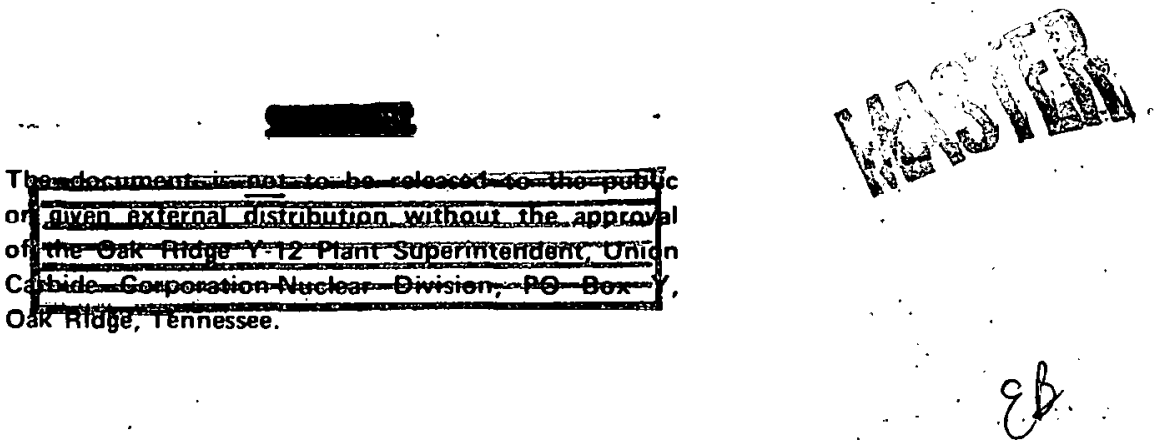

DISTRIBUTION OF IHIS OOCUMENTT IS IINLIMITEO 
DISTRIBUTION

Atomic Energy Commission-ORO

Keller, C. A.

Zachry, D. S. Jr

Dow-Rocky Flats

Hartz, S. J.

Lawrence Livermore Laboratory

Liptai, R. G.

Tatro, C. A.

Los Alamos. Scientific Laboratory

Elliot, D. E.

Oak Ridge Gaseous Diffusion Plant.

Jordan, R. G.

Wilcox, W. J., Jr

Oak Ridge $Y-12$ Plant

Bernander, N. K.

Blake, H. W.

Briscoe, O.W.

Burditt, R. B.

Burkhart, L. E.

Cook, J. L.

Courtright, E. L.

Davenport, C. M.

Denny, A. (2)

Dritt, W. S.

Gerth, H. L.

Hemphill, L.F.
Jackson, V.C.

Jones, F.W.

Kahl, K. G.

Keith, Alvin

Knight, C. E.

Mason, D. L.

McLendon, J.D.

Mitchel, G. W.

Moyer, M. W.

Snow, S. G.

Stephens, A. E.

Thompson, J. $\mathrm{H}$.

Wallace, S. A.

Weathersby, W. E.

Wesley, R. L.

Wright, C. C.

Yaggi, W. J.

Y- 12 Central.Files (5)

$Y-12$. Central Files (master copy)

$Y-12$ Central Files (route)

$Y-12$ Central Files ( $Y-12 R C)$

Paduçah Gaseous Diffusion Plant

Winkel, R. A.

Sandia-Albuquerque

Ballard, D. W.

Burchette, O. N., Jr

Sandia-Livermore

Baker, A. F.

Beattie, A. G.

Hiles, L. A.

Rathbun, D. K. 


\section{ABSTRACT}

An analytical solution for acoustic flaw triangulation on thin spherical shells has been prepared. A FORTRAN program is provided which takes as input the differences in the arrival times of acoustic energy at the transducers and evaluates the spherical coordinates of the flaw. Only three experimental time differences need be measured. For this measurement, four transducers are required. One transducer is placed at $(R, 0,0)$ in spherical coordinates; the other three are spaced 90 degrees apart around the equator. 


\section{CONTENTS}

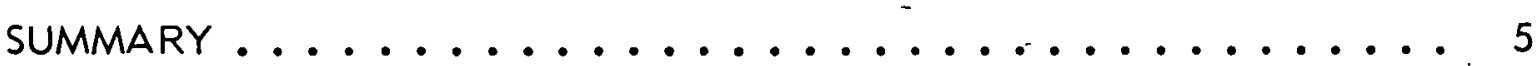

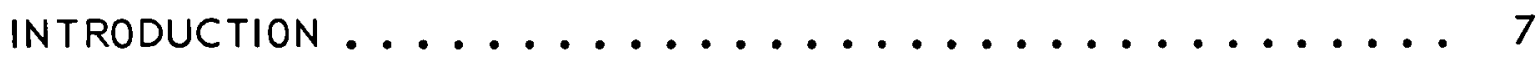

ANALYTICAL SOLUTION OF THE TRIANGULATION PROBLEM ......

Mathematical Analysis. ......................

Computer Simulation. ....................... 14

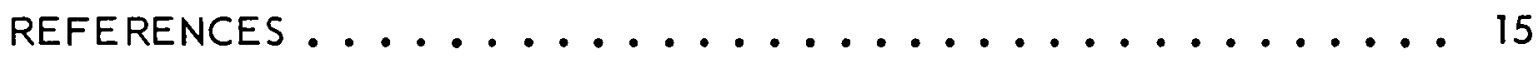

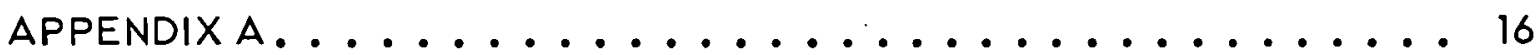

Acoustic Emission Simulation .................. 16

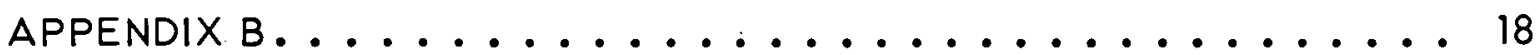

Solution of the Triangulation Equations for Transducer Array $(1,2,3) \ldots 18$

APPENDIX C............................... 19

Flow Chart for the Triangulation Algorithm. . . . . . . . . . 19

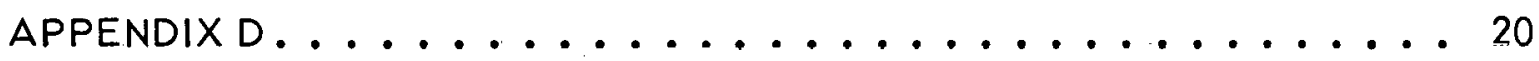

Listing of the Computer Program . . . . . . . . . . . . 20

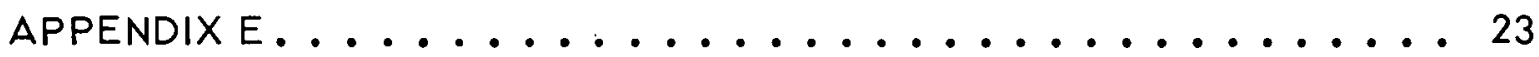

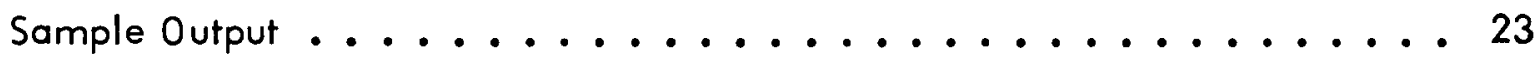

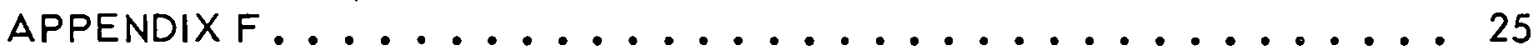

Other Solutions Using the Four-Transducer Array . . . . . . . . . . 25 


\section{SUMMARY}

The problem of acoustic flaw triangulation on a thin spherical shell has been solved analytically for one particular arrangement of acoustic emission transducers. If four transducers are used, one placed at a pole and the other three 90 degrees apart around the equator, then the flaw coordinates $\theta$ and $\varphi$ are given by:

$$
\begin{gathered}
\cot \varphi=-\frac{(\sin b \cdot \cos b+\sin c \cdot \cos c) \pm \sqrt{2 \cos b \cdot \cos c \cdot \cos (b-c)}}{\cos ^{2} b+\cos ^{2} c}, \\
\cot \varphi=-\frac{(\sin c \cdot \cos c+\sin d \cdot \cos d) \pm \sqrt{2 \cos c \cdot \cos d \cdot \cos (d-c)}}{\cos ^{2} c+\cos ^{2} d}, \\
\sin \theta=\cos c \cdot \cot \varphi+\sin c, \text { and } \\
\cos \theta=\cos b \cdot \cot \varphi+\sin b .
\end{gathered}
$$

In these equations:

$$
\begin{gathered}
b=v \Delta t_{13} / R, \\
c=v \Delta t_{23} / R, \text { and } \\
d=v \Delta t_{34} / R,
\end{gathered}
$$

where:

$\checkmark$ represents the speed of sound in the shell material (assumed constant and of a single value),

$R$ The mean radius of the spherical shell,

$$
\begin{gathered}
\Delta t_{13}=t_{3}-t_{1}, \\
\Delta t_{23}=t_{3}-t_{2} \text {, and } \\
\Delta t_{34}=t_{3}-t_{4} .
\end{gathered}
$$

The time of arrival at transducer (i) of the acoustic emission energy from the flaw site would be represented by $t_{i}$. The correct value of $\cot \varphi$ is given by that combination of signs which yields equality between the two formulas for $\cot \varphi$.

It will be noted that only three experimental values $\left(\Delta t_{13}, \Delta t_{23}, \Delta t_{34}\right)$ are required to locate a flaw site, even though four transducers are used. This apparent discrepancy is because the time differences from the three transducers located mutually 
90 degrees apart on a spherical shell are not all independent. Any one difference can be expressed as a linear combination of the other two.

A FORTRAN program has been written to test the results just described. 


\section{INTRODUCTION}

For some time, acoustic emission monitoring has been recognized as a promising technique for evaluating the integrity of structures that fail catastrophically due to flaw formation and growth or to other forms of accumulative damage. Such is the case with various metal pressure vessels, boilers, and high-pressure piping systems as well as with complex structures such as filament-wound reinforced composites. The distinct advantage of this technique is that the flaw supplies the signal for detection and thus, much like an earthquake, provides valuable information regarding size, orientation, and location of the source of emission. Moreover, if the sound propagation characteristics of the structure are known, the use of multiple receiving transducers can provide the necessary information to locate and characterize flaws. The success of this technique naturally requires that the flaw type and its severity be correlated to its characteristic emission pattern.

Triangulation techniques have been devised for nuclear vessels and piping systems, and digital programs are now available which solve the triangulation equations for cylindrical (1) and spherical(2) shapes. These programs are quite general and allow the transducers to be placed on piping external to the primary pressure vessels as well as anywhere on the vessel itself. Due to the complex geometry, the triangulation equations are solved by iteration and the programs are necessarily complex. For situations that allow freedom of placement of the transducers on the shell, the receiving points can be selected so as to simplify the equations and thus allow a closed-form solution. This latter approach is taken here and the equations are solved for spherical shapes.

Since one of the objectives of this study was to develop a technique suitable for structures that might have many flaw sites, a unique triangulation scheme was required. It was felt that the use of existing iterative programs would result in excessive total computation times when many flaws are involved. Also, the lack of uniqueness in the available solutions would require additional data to eliminate the "phantom" solutions. Thus, the objectives of this work were to obtain a unique solution of simple form that would require a minimum number of transducers and a minor amount of machine computation time. The computer program is provided for use whenever the number of flaws is so large as to make hand calculation impractical.

It must be emphasized that any triangulation scheme is approximate due to the assumptions that are made to allow a simple solution to a basically complicated problem. The effects of each approximation on the actual measurements should be determined if the precision of the technique is to be truly evaluated. Unfortunately, this approach would be time consuming due to the many factors that would have to be considered. An alternate approach is to triangulate on a known flaw location and thus compare measured and actual positions. In situations when the introduction of actual flaws is impossible, the acoustic source can be simulated using focused 
ultrasonic pulsers or other similar devices. (3) Results using this approach on a spherical part are given in Appendix A.

For the sake of analysis, the following assumptions were made regarding the propagation and reception of the acoustic flaw signal:

1. The sound is propagated in a single mode and a unique speed of sound propagation is assigned to the structure.

2. The receiving transducers are infinitely small.

3. The portion of the flaw producing the signal is negligibly small.

4. Each burst of sound arrives at all the receiving points.

5. Erroneous time-difference measurements caused by overlapping pulses and/or multiple flaws are negligible.

6. Time slewing errors, due to differences in interchannel response times or multitrack recording and playback, are negligible.

The following assumptions are particular to the spherical shape:

1. The shell is perfectly spherical with an infinitely thin wall.

2. Sound propagates according to the geodesic (great-circle paths).

3. Timing errors due to reflections within the shell can be reduced by using the mean radius or some other correction factor. 


\section{ANALYTICAL SOLUTION OF THE TRIANGULATION PROBLEM}

\section{MATHEMATICAL ANALYSIS}

Two major needs must be satisfied in an exact analysis of the problem. One is the selection of the number and placement of the transducers; the other is the solution of the system of triangulation equations in such a way that uniqueness is assured. The approach taken here was to try the simplest arrangement possible. Spherical coordinates were used throughout. A superficial analysis shows that at least three, but possibly more, transducers are required, so that the analys is was started with three transducers placed mutually 90 degrees apart, as indicated in Figure 1. Figure 1 also defines some mathematical quantities used in the derivation that follows.

Assuming a flaw site (A) with spherical coordinates $(R, \theta, \varphi)$ located by the vector, $\vec{f}$, the angles $\theta_{1}, \theta_{2}, \theta_{3}$ can be calculated between $\vec{f}$ and the $X, Y, Z$ axes, respectively. However, it is the inverse problem that is of interest here: Given $\theta_{1}, \theta_{2}, \theta_{3}$; calculate $R, \theta, \varphi$.

The experimentally measurable quantities will be:

$$
\begin{gathered}
\Delta t_{12}=t_{2}-t_{1} ! \\
\Delta t_{23}=t_{3}-t_{2}, \text { and } \\
\Delta t_{13}=t_{3}-t_{1},
\end{gathered}
$$

where $t_{i}$ represents the time of arrival at transducer (i) of acoustic energy from the flaw site, measured from an arbitrary time base. Evidently:

$$
\Delta t_{23}=\Delta t_{13}-\Delta t_{12},
$$

so that only two measurements are independent. Seemingly, this number should be enough because solutions are needed for only two coordinates: $\theta$ and $\varphi$. Referring to Figure I, if the "S" lines represent great-circle distances, then:

$$
\begin{gathered}
S_{2}-S_{1}=R\left(\theta_{2}-\theta_{1}\right)=v \Delta t_{12} ; \\
S_{3}-S_{2}=R\left(\theta_{3}-\theta_{2}\right)=v \Delta t_{23}, \text { and } \\
S_{3}-S_{1}=R\left(\theta_{3}-\theta_{1}\right)=v \Delta t_{13} .
\end{gathered}
$$

Letting:

$$
a=\frac{v}{R} \Delta+12,
$$




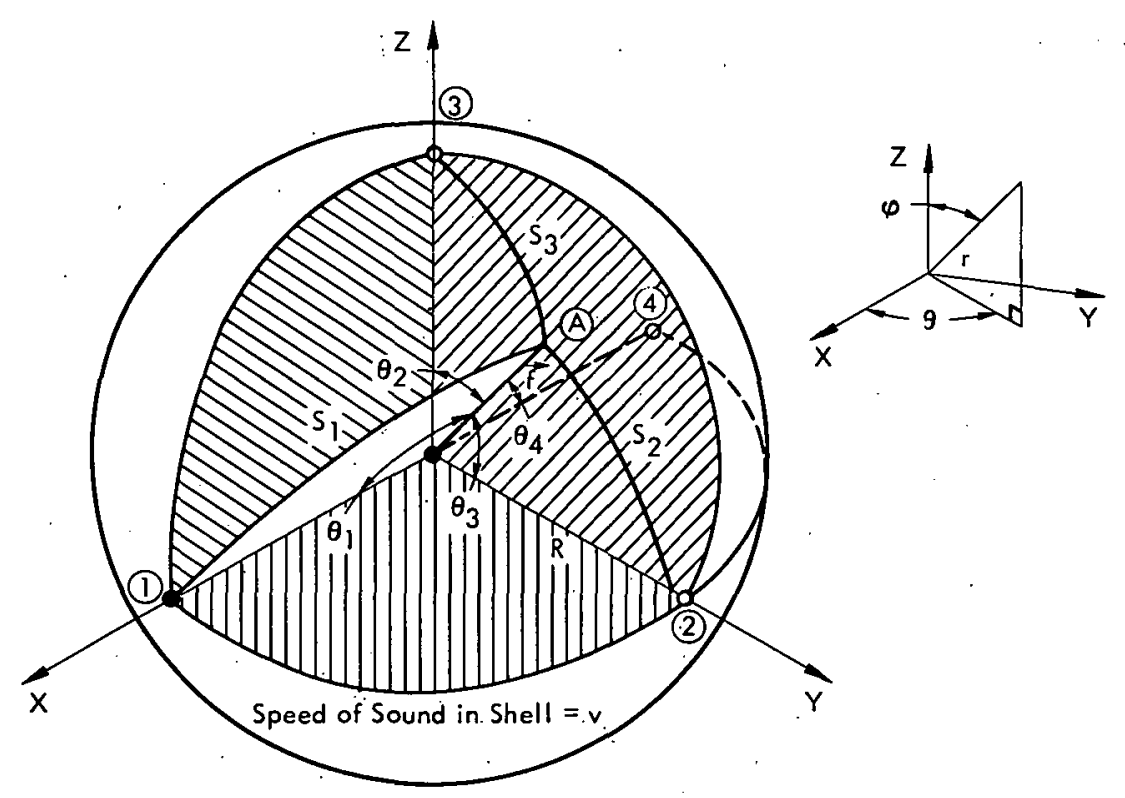

Figure 1. GEOMETRIC AND MATHEMATICAL QUANTITIES.

$$
\begin{gathered}
b=\frac{v}{R} \Delta t_{13}, \text { and } \\
c=\frac{v}{R} \Delta t_{23},
\end{gathered}
$$

then:

$$
\begin{gathered}
\theta_{2}-\theta_{1}=a, \\
\theta_{3}-\theta_{1}=b, \text { and } \\
\theta_{3}-\theta_{2}=c .
\end{gathered}
$$

The cosines of the angles $\theta_{1}, \theta_{2}, \theta_{3}$ are direction cosines for the flaw vector, $\overrightarrow{f ;}$ hence, the preceding set of equations (4) become:

$$
\begin{gathered}
\cos ^{-1}(\sin \varphi \cdot \sin \theta)-\cos ^{-1}(\sin \varphi \cdot \cos \theta)=a, \\
\varphi-\cos ^{-1}(\sin \varphi \cdot \cos \theta)^{\circ}=b \cdot, \text { and } \\
\varphi-\cos ^{-1}(\sin \varphi \cdot \sin \theta)=c,
\end{gathered}
$$

one of which is redundant because, from Equation 2, $a=b-c$. If the last two are solved for $\theta$ and $\varphi$, the solution is: 


$$
\begin{gathered}
\cot \varphi=-\frac{(\sin b \cdot \cos b+\sin c \cdot \cos c) \pm \sqrt{2 \cos b \cdot \cos c \cdot \cos (b-c)}}{\cos ^{2} b+\cos ^{2} c}, \\
\sin \theta=\cos c \cdot \cot \varphi+\sin c, \text { and } \\
\cos \theta=\cos b \cdot \cot \varphi+\sin b,
\end{gathered}
$$

as shown in Appendix B.

But, now, a peculiar difficulty arises in using the solution of these equations (6). Direct substitution of a large number of test cases showed that in the $\cot \varphi$ equation, the positive $(+)$ sign is used for flaws in one region on the sphere and the negative $(-)$ sign is used. in the other. These two regions are separated by the curve:

$$
\sqrt{2 \cos b \cdot \cos c \cdot \cos (b-c)}=0 \text {, }
$$

or:

$$
\cos a \cdot \cos b \cdot \cos c=0 \text {. }
$$

This curve and the corresponding regions are symmetrical about the line joining $\left[R, \pi / 4, \cos ^{-1}(1 / \sqrt{3})\right]$ and $\left[R, 5 \pi / 4, \cos ^{-1}(1 / \sqrt{3})\right]$. Figure 2 points out these relationships.

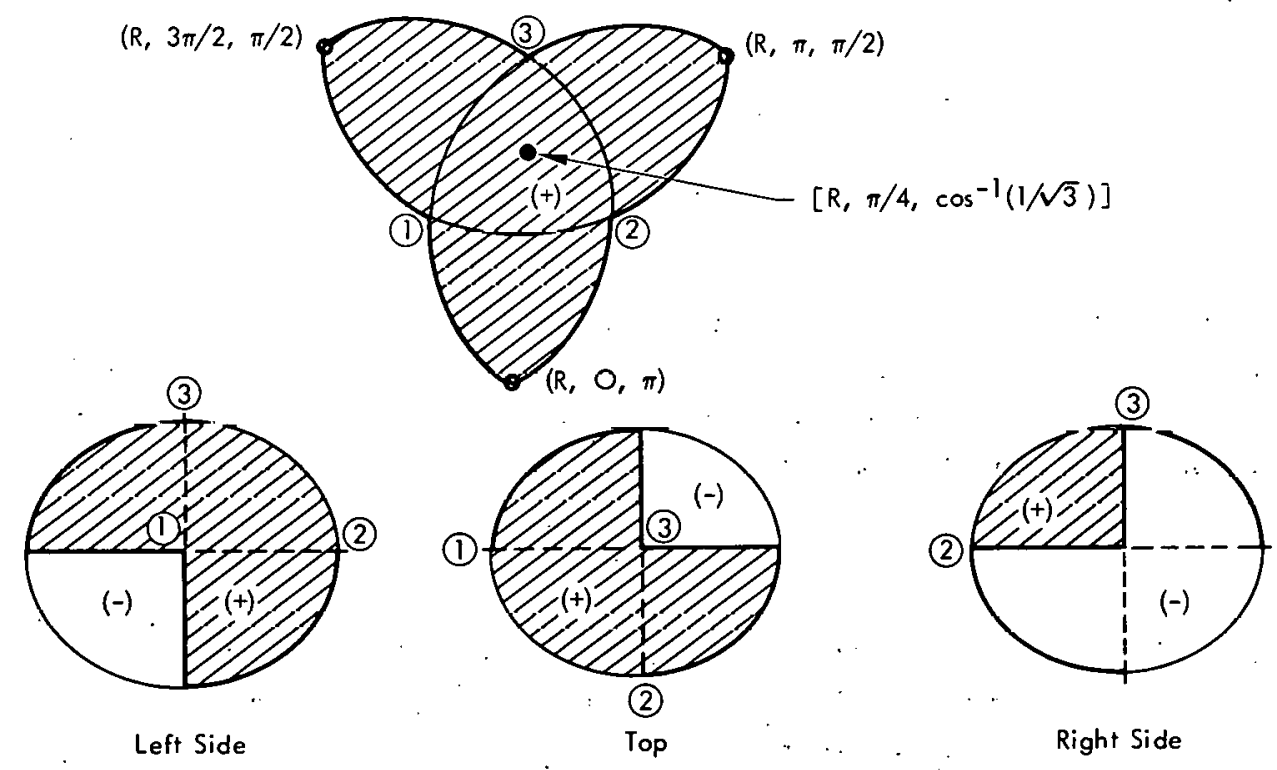

Figure 2. SIGN INDÉTERMINACY ARISING IN A THREE-TRANSDUCER ARRANGEMENT.

If a pair $(b, c)$ is put into the (6) equations, one sign will give the correct values for $(\theta, \varphi)$ in one region and the other sign will give an extraneous, but valid, 
solution in the other region. Finding the correct solution, then, hinges upon the ability to determine which region contains the flaw. Since at this point $b$ and $c$ are the only independent bits of information given, it seems unlikely that the problem can be resolved. Some new, independent condition must be supplied; and, since only time differences can be measured, at least one more transducer is needed.

Correct placement of a fourth transducer, so that the whole sphere was completely resolved, required a close study to fix the relationship between a correct solution and its corresponding extraneous solution. It was observed that the sign combinations on $a, b$, and $c$ were constant in six equal "orange slice" sectors symmetrical about the line joining $\left[R, \pi / 4, \cos ^{-1}(1 / \sqrt{3})\right]$ and $\left[R, 5 \pi / 4, \cos ^{-1}(1 / \sqrt{3})\right]$, as seen in Figure 3. Each "orange slice", or sector, is divided into two equal and antisymmetrical parts by the curve bounding the two sign regions of Figure 2. It then follows that both solutions for a given $b$ and $c$ must lie in the same sector on opposite sides of the diagonal. This fact was the bas is for the final solution outlined in the development that follows.

A fourth transducer was placed at $(R, \pi, \pi / 2)$, as given in Figure 1 . This fourth transducer was used with Transducers 2 and 3 to make another three-transducer system analogous to the first, but rotated +90 degrees about the $Z$ axis. If the experimental value, $d$, in the new system corresponds to $c$ in the old, then it follows that:

$$
\cot \varphi=-\frac{(\sin c \cdot \cos c+\sin d \cdot \cos d) \pm \sqrt{2 \cos c \cdot \cos d \cdot \cos (d-c)}}{\cos ^{2} c+\cos ^{2} d} .
$$

This equation must give the same value for $\varphi$ as the corresponding equation in the (6) group because the $\varphi$ coordinate is unchanged in the rotated system. The extraneous solution from this equation, however, will not be the same as the extraneous solution from the (6) equation. The situation is shown diagrammatically in Figure 4.

The orange-slice sector containing the real solution in the rotated system is always aligned so that only one or the other of its possible sign regions overlaps the solution sector in the original system. Hence, of the four possible solutions from the $\cot \varphi$ equations $[(6)$ and (8)], only two will be equal-the two being from separate equations. It then follows that the correct sign in the (6) equation can be chosen by cross checking with the (8) equation until a match is made. The success of the method depends upon the fact that both sign regions of the rotated sector cannot overlap parts of both sign regions in the original sector, as indicated in Figure 4. 


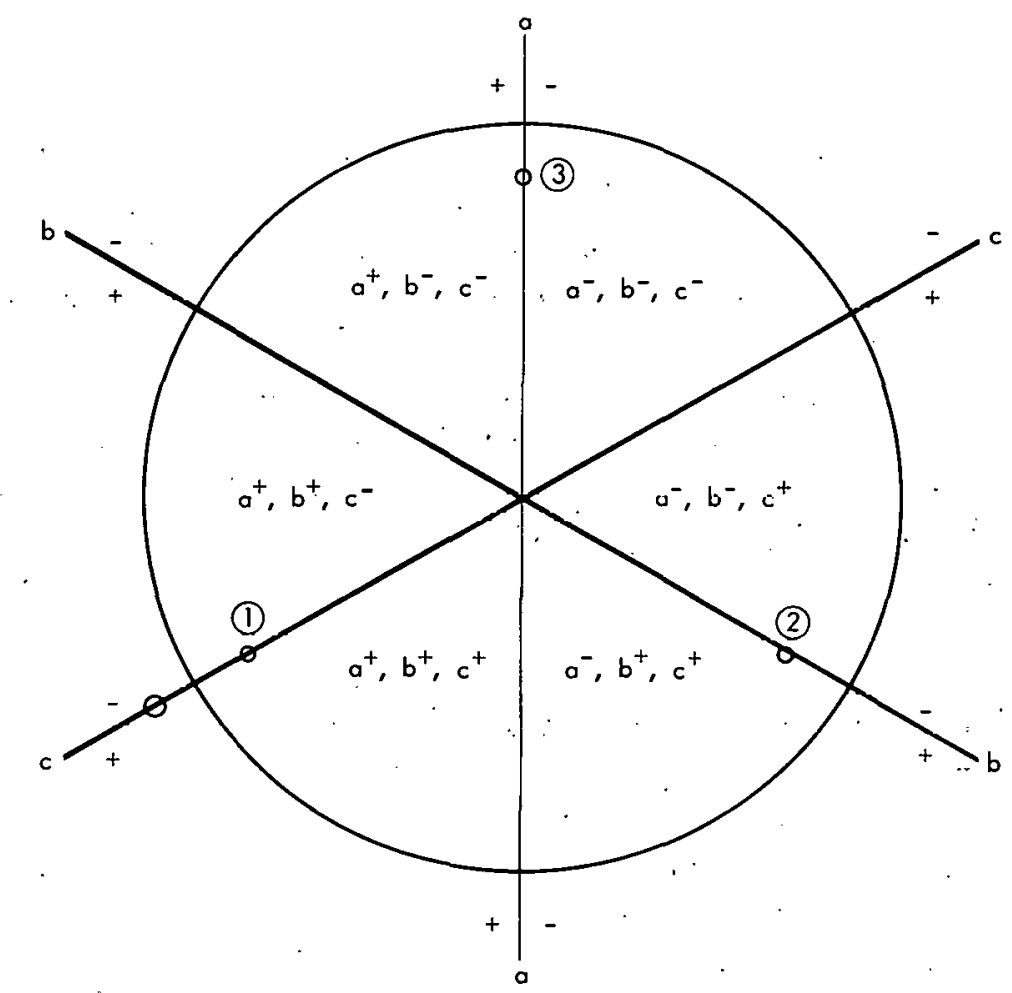

Figure 3. TILTED VIEW OF THE SPHERE SHOWING THE REGIONS OF DISTINCT SIGN COMBINATIONS.

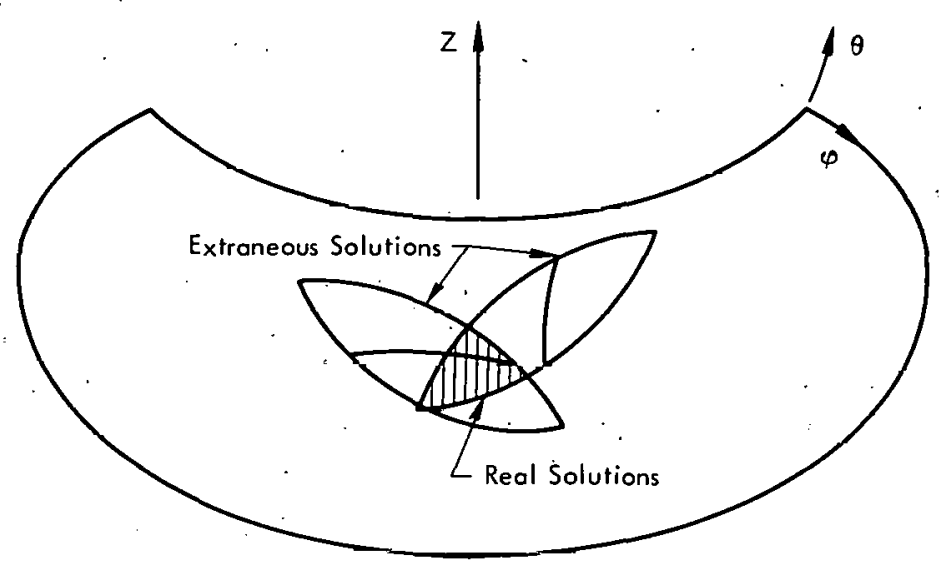

Figure 4. OVERLAPPING OF THE SIGN SECTORS BETWEEN THE ORIGIINAL AIND ROTATED SYSTLMS OF TRANISDUCERS.

The basis for calling this method "triangulation", even though four transducers are involved, is that only three distinct time differences $(b, c$, and $d$ ) are used in calculating the flaw coordinates. 


\section{COMPUTER SIMULATION}

An algorithm was worked out for the unique solution for flaw coordinates given only $b, c$, and $d$, and the (6) and (8) equations. Appendix $C$ gives the flow chart. Basically, the procedure is as follows: There are two equations for $\cot \varphi$, both having a sign option. One equation is in terms of $b$ and $c$; the other in terms of $d$ and $c$. In either equation, a zero denominator indicates the special case of a flaw at one or the other of the poles. Therefore, a preliminary check tests the denominators for zero; and, if either is zero, the quantity, $b$, is tested to indicate which pole is involved. Due to measurement error and finite-sized flaw sites, a flaw at either pole may not yield $b, c$, and $d$ values which make the denominators exactly zero; hence, the test is made not for exactly zero but for some very small range about zero.

For the case of the nonzero denominators, all four possible values of $\cot \varphi$ are calculated and compared. The sign option in the first equation, which most nearly yields exact agreement with either sign option in the second equation, is accepted as correct. Again, this procedure is necessary because of possible small errors in measuring $b, c$, and $d$. Once the sign option is decided, $\theta$ and $\varphi$ are calculated from the (6) equation, using inverse trigonometric functions supplied in the computer. Principal values calculated from these inverse functions are modified so as to have the range of the experimental coordinates.

A FORTRAN program was written to test the algorithm. A listing is given in Appendix D. The program was written for the CDC-6400 time-share computer and uses standard FORTRAN statements, with the exception of the inverse cosine function which is heavily used. The program takes input coordinates $\theta$ and $\varphi$ and generates simulated values ( $a, b, c$, and $d)$, and then processes $b, c$, and $d$ as if they were experimental values. The output coordinates must then be equal to the input values. The comparison is printed out. The output values of $a, b, c$, and $d$ are calculated from the output values of $\theta$ and $\varphi$ for comparison with the input. This step is of little value where very accurate inputs are simulated, but could be useful where inputs with experimental errors are used. The program can be converted for data processing simply by removing the segment which simulates the $a, b, c$, and $d$ inputs and adding a segment to directly input these as experimental quantities. Appendix $E$ shows a sample output for simulated flaws every 45 degrees on either coordinate. 


\section{REFERENCES}

(1) Waite, E. V. and Moore, K. V.; Acoust-A Digital Program for Acoustic Triangulation of Nuclear Vessels, IDO-17280; Atomic Energy Commission Research and Development Report, Phillips Petroleum Company; November 1968.

(2) Waite, E. V.; Acoust-S-A Digital Program for Acoustic Triangulation of Spherical Vessels; IN-1369; Atomic Energy Commission Research and Development Report, Idaho Nuclear: Corporation; March 1970:

(3) Fowler, K. A.; "Acoustic Emission Simulation Test Set," Materials Research and Standards, 11, (3) p 35; March 1971.

(4) Rathbun, D.K., Beattie, A. G., and Hiles, L. A.; Filament Wound Materials Evaluation with Acoustic Emission, SCL-DC-70-260; Sandia Laboratories Report, Sandia Laboratories, Livermore, California; April 1971. 


\section{APPENDIX A}

\section{ACOUSTIC EMISSION SIMULATION}

Acoustic emission simulation was used on a three-inch-diameter, filament-wound, reinforced, composite shell in order to test the triangulation scheme. Four receiving transducers were bonded to the vessel in an orthogonal array, as shown in Figure $A-1$. Acoustic emission simulating a flaw was produced by pulsing a fifth transducer having a solid exponential lens to concentrate the sound at the desired location. The receiving transducers were PZT ceramic crystals with approximately a $140-\mathrm{kHz}$ resonant frequency. A $1-\mathrm{MHz}$ resonant PZT crystal was used as the pulser. The sound propagation velocity was measured by transmitting and receiving with two identical focused transducers positioned 180 degrees apart on the sphere. The propagation time due to the exponential lenses was determined by placing these two transducers in contact. The "angular speed of sound" $(V / R)$ was measured to be $5.4 \times 10^{4}$ per sec and the propagation time for 180 degrees on the part was approximately 58 microseconds. The speed of sound was nearly half that of the published(4) longitudinal mode value for similar parts. It is likely that the shear wave was the predominant mode of propagation in these tests.

The pulser was placed at the position $\theta=45$ degrees and $\varphi=90$ degrees, and the following time differences were measured using a time interval meter and an oscilloscope:

$$
\begin{gathered}
t_{3}-t_{1}=15 \mu \mathrm{sec}, \\
t_{3}-t_{1}=15 \mu \mathrm{sec}, \text { and } \\
t_{3}-t_{4}=-14 \mu \mathrm{sec} .
\end{gathered}
$$

These values represent the averages of many readings and have been rounded off to the nearest microsecond. Low-level signals preceding the main pulse, likely due to the longitudinal wave, result in at least a $1-\mu \mathrm{sec}$ uncertainty. The corresponding values of $b, c$, and $d$ were:

$$
\begin{gathered}
b=0.81 \mathrm{rad}=46.4 \text { degrees, } \\
c=0.81 \mathrm{rad}=46.4 \text { degrees, and } \\
d=-0.76 \mathrm{rad}=-43.3 \text { degrees } .
\end{gathered}
$$

Calculated values of $\theta$ and $\varphi$ were:

$$
\begin{gathered}
\theta=45.0 \text { degrees, and } \\
\varphi=91.4 \text { degrees, }
\end{gathered}
$$




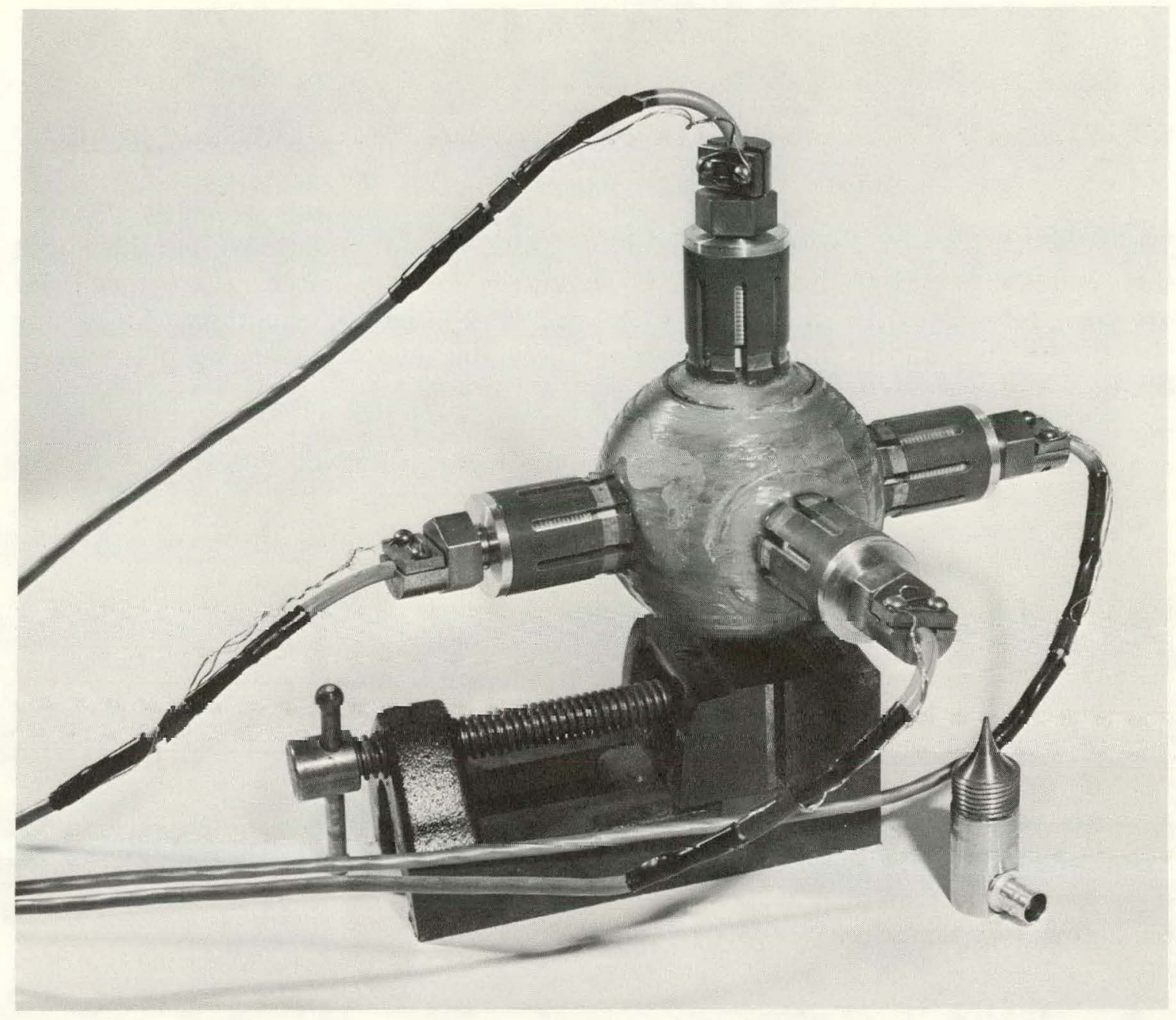

140425

Figure A-1. SPHERICAL SHELL WITH TRANSDUCERS.

which are in reasonable agreement with the actual pulser coordinates. For this work, the sensitive area of each receiving transducer was $0.5 \mathrm{inch}$; and, thus, the part size-to-crystal diameter ratio was approximately six. It could be expected that the precision of the technique would increase with this ratio, provided that the part diameter-to-wall thickness ratio remains constant. 


\section{$\underline{\text { APPENDIX B }}$}

SOLUTION OF THE TRIANGULATION EQUATIONS FOR TRANSDUCER ARRAY $(1,2,3)$

The quantities $a, b$, and $c$ are calculated using the (3) equations, provided that the "angular velocity" of sound $(V / R)$ is known for the vessel. These quantities are then related to the flaw coordinates $(\theta, \varphi)$ by the $(5)$ equations. Since any two of the (5) equations are independent, the last two are selected for ease of solution and are rewritten as:

$$
\begin{aligned}
& \cos (\varphi-b)=\cos \varphi \cdot \cos b+\sin \varphi \cdot \sin b=\sin \varphi \cdot \cos \theta, \text { and } \\
& \cos (\varphi-c)=\cos \varphi \cdot \cos c+\sin \varphi \cdot \sin c=\sin \varphi \cdot \sin \theta .
\end{aligned}
$$

Division of both equations by $\sin \varphi$ yields:

$$
\begin{aligned}
& \cot \varphi \cdot \cos b+\sin b=\cos \theta \text {, and } \\
& \cot \varphi \cdot \cos c+\sin c=\sin \theta .
\end{aligned}
$$

A single equation for $\cot \varphi$ results from squaring the (10) equations and adding. Solution of this last equation by the quadratic formula yields the first of the (6) equations for determining $\varphi$. The calculated value of $\cot \varphi$ is then used in the remaining two equations to determine $\theta$. 


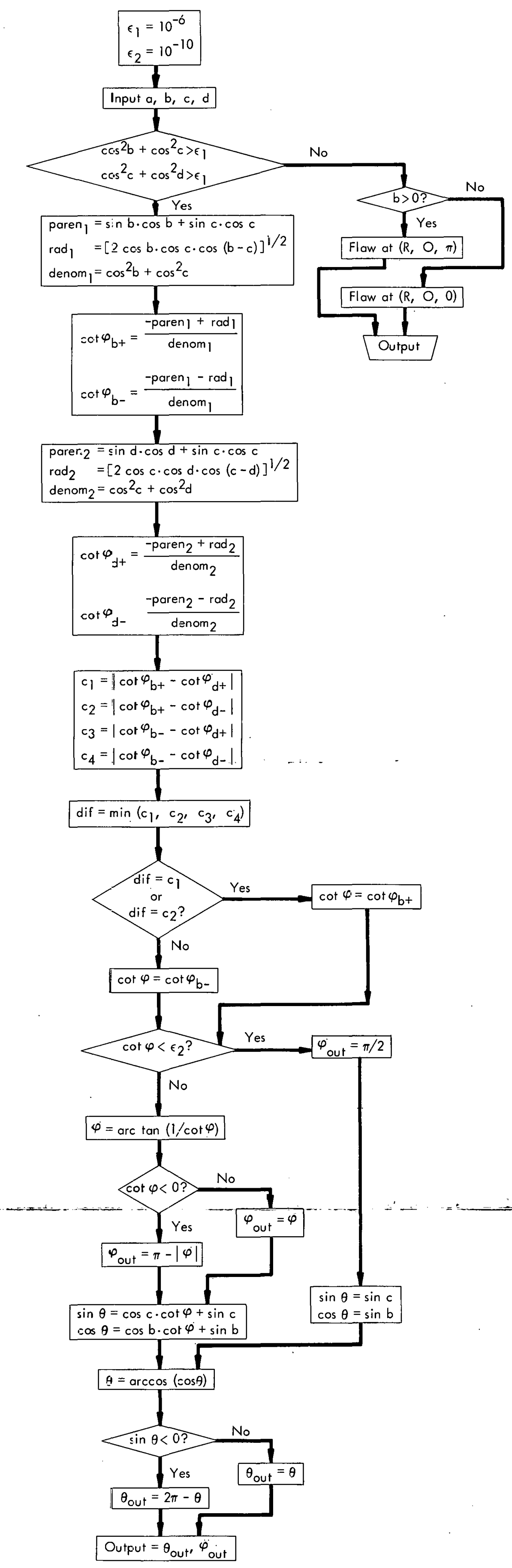

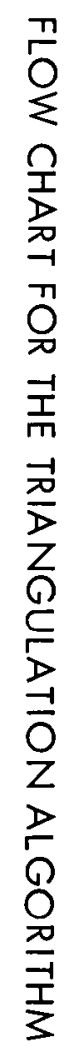




\section{APPENDIX D}

\section{LISTING OF THE COMPUTER PROGRAM}

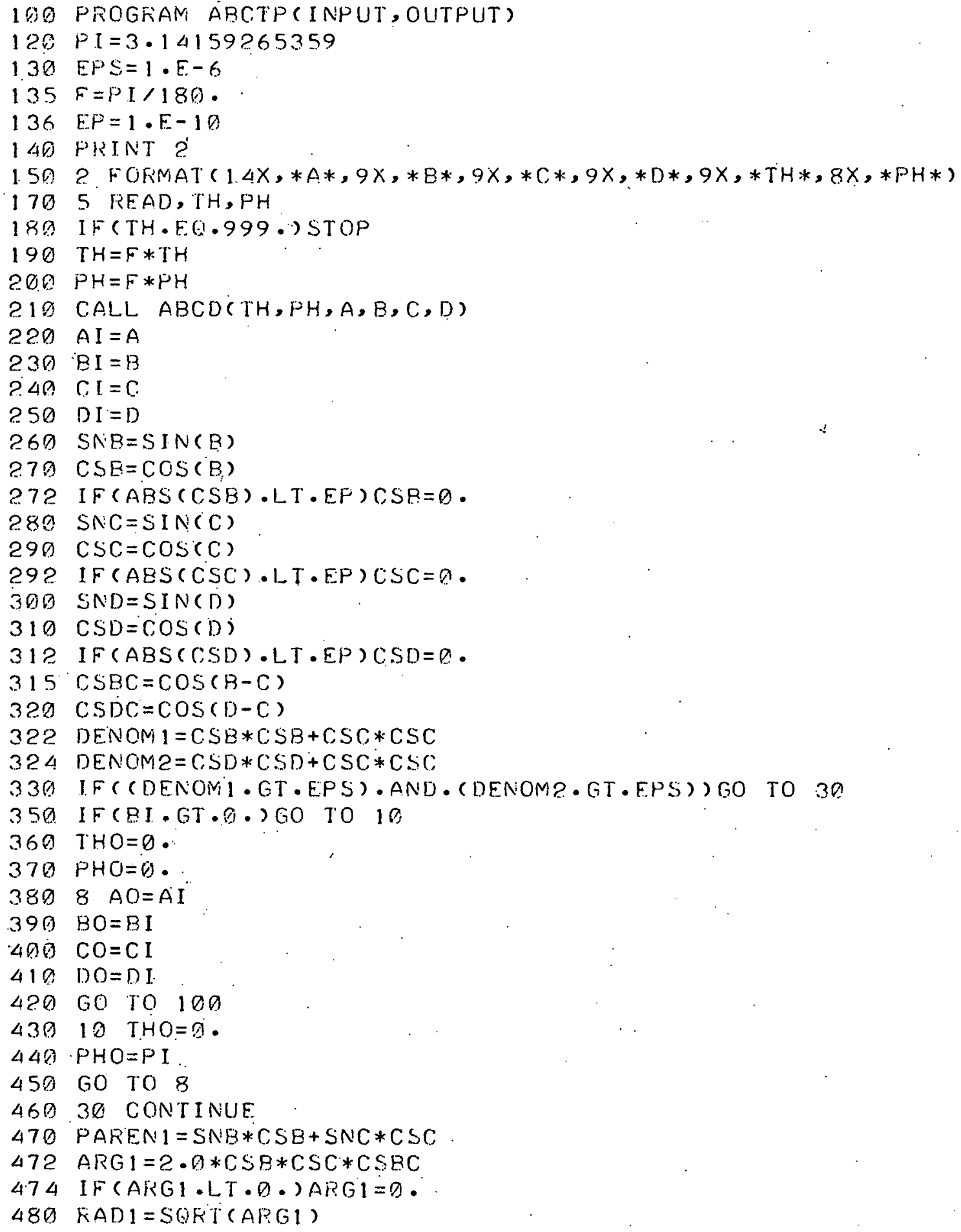




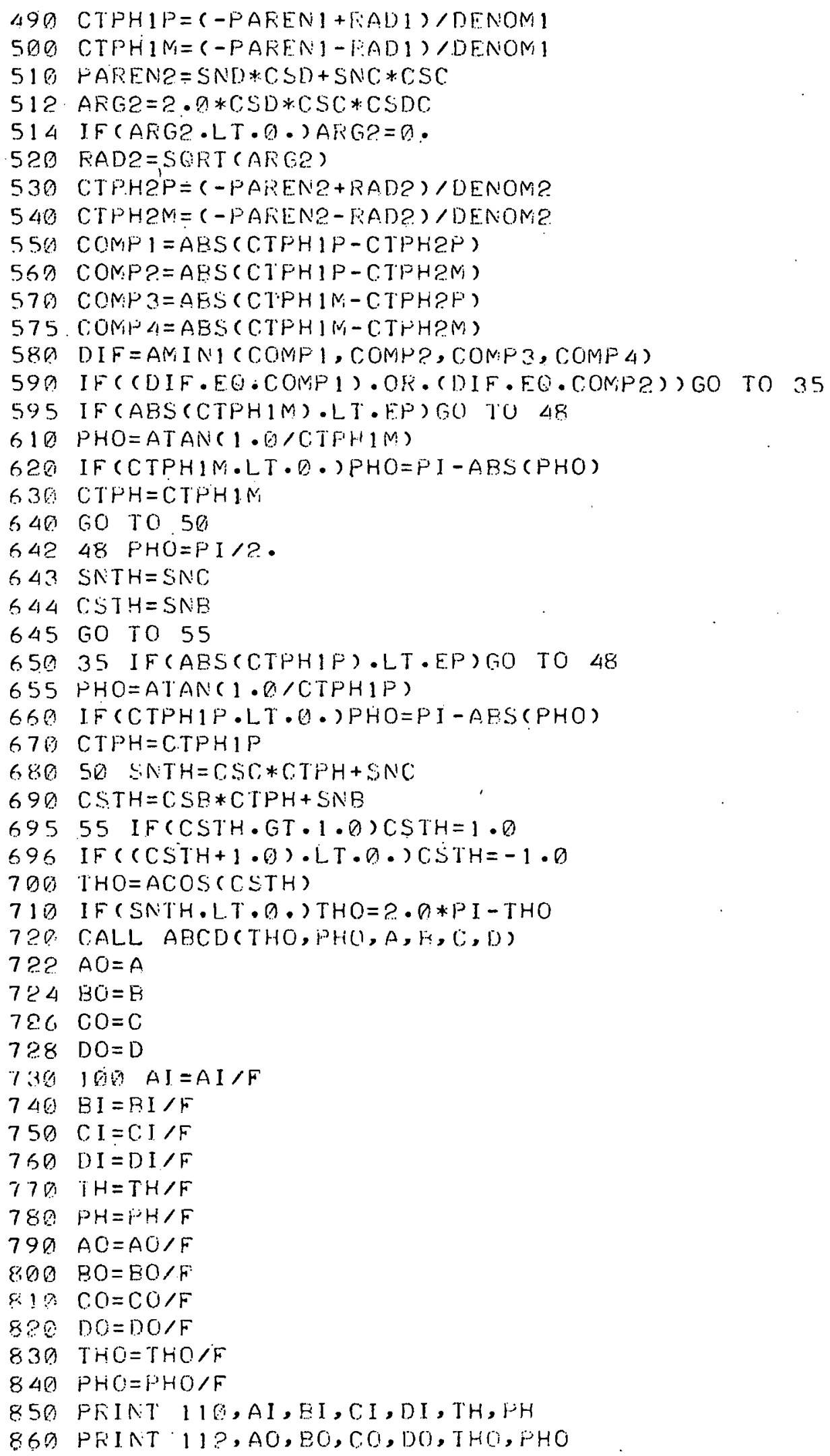




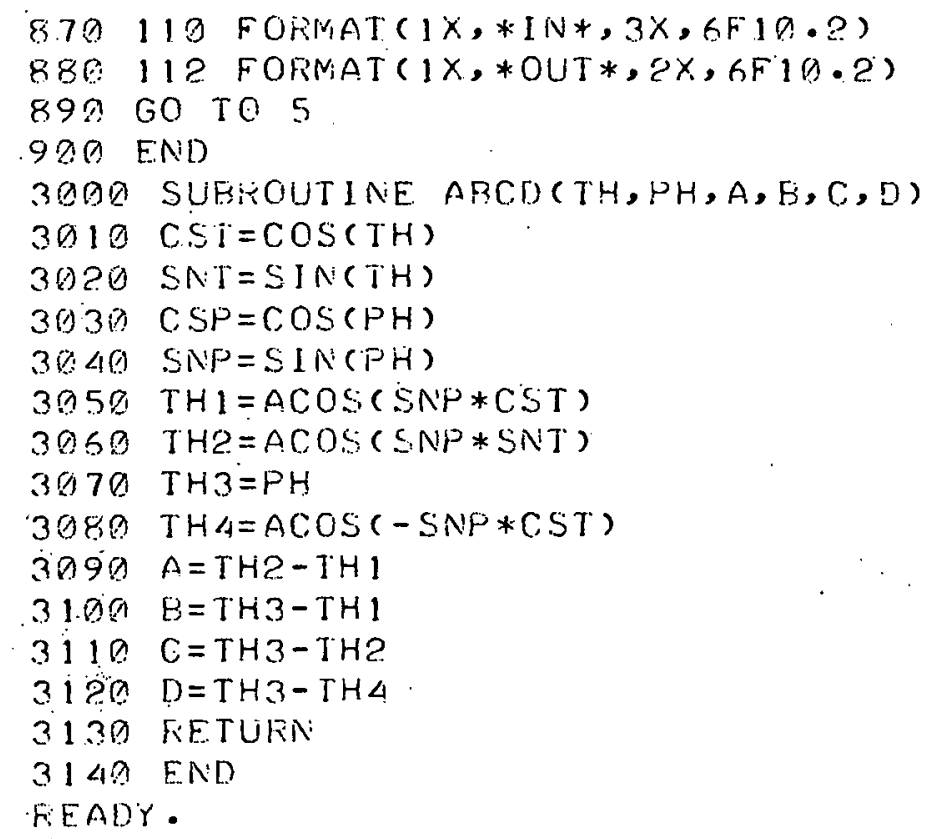




\section{APPENDIX E}

\section{SAMPLE OUTPUT}

$71 / 06 / 03 \cdot 09 \cdot 50 \cdot 12$.
PFOGRAM ABCTP

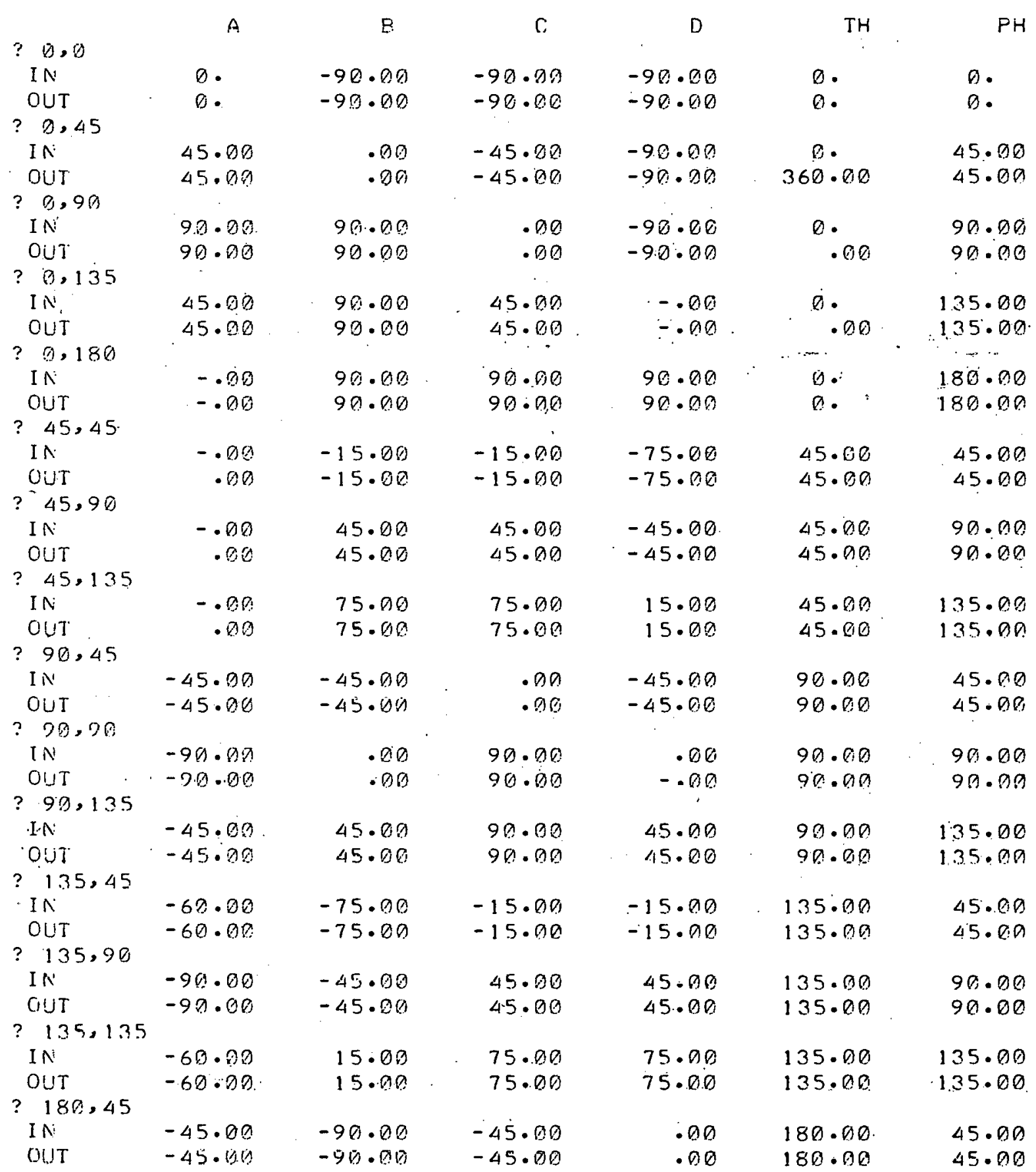




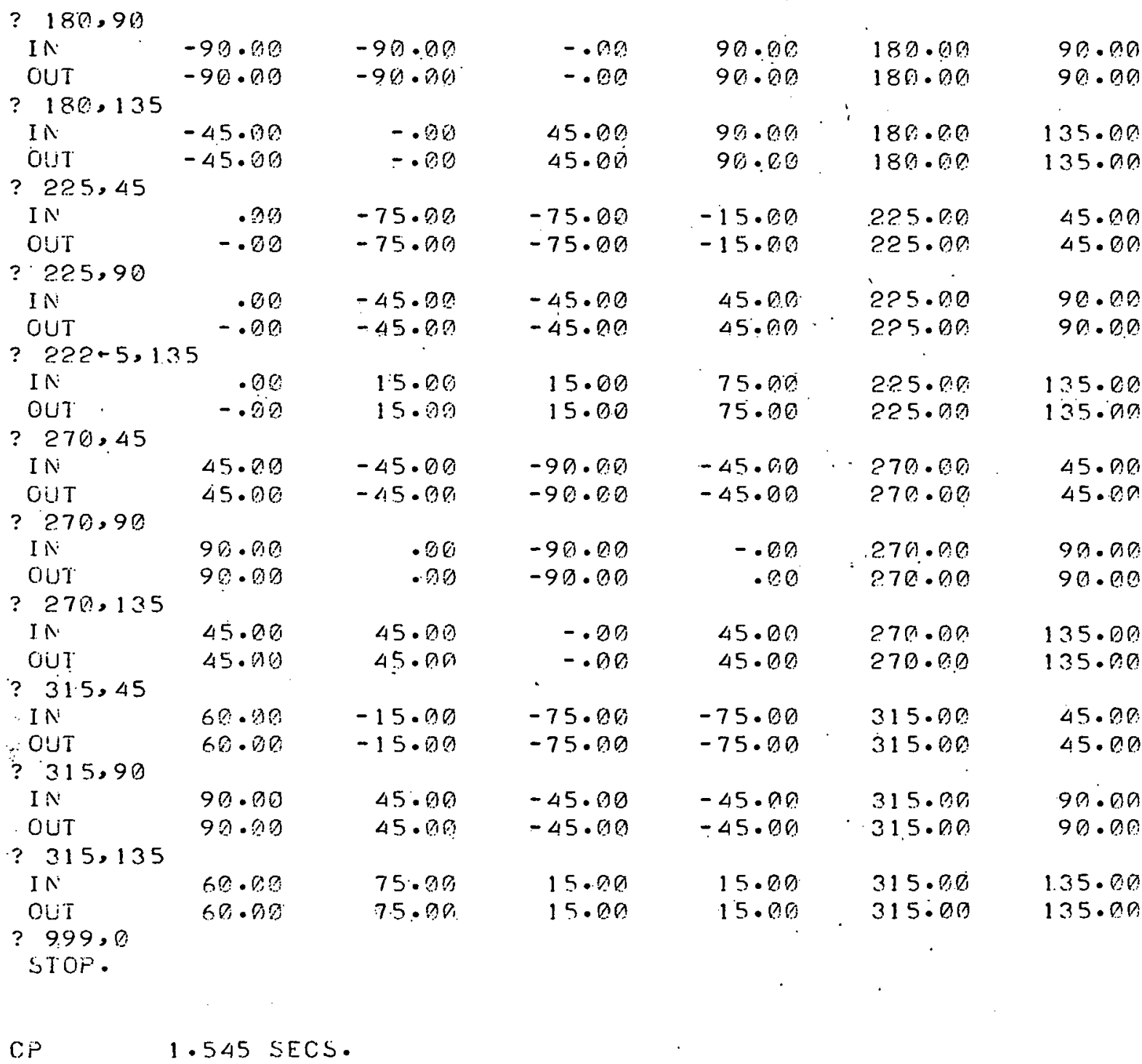

RUN CONPLETE. 


\section{APPENDIX F}

\section{OTHER SOLUTIONS USING THE FOUR-TRANSDUCER ARRAY}

The computer program of Appendix $C$ calculates the flaw coordinates $(\theta, \varphi)$ using the experimentally measured time differences $t_{3}-t_{1}$ and $t_{3}-t_{2}$. The time difference, $t_{3}-t_{4}$, involving the fourth transducer, is required only to select the correct flaw site from the two possible solutions to the problem. This approach places the entire burden of establishing flaw location on Transducers 1, 2, and 3, and assumes that the measured time differences for these receivers are perfectly accurate. If, instead, equal importance is placed on time measurements from all four transducers, the triangulation problem is overdetermined and the solution can be obtained in a variety of ways. All these solutions would indicate the same flaw location provided that the time-difference measurements were completely accurate. However, because of timing errors due to wave-mode conversion and the finite thickness of the vessel wall, the various solutions will not quite agree. By solving for all or part of the flaw sites, an indication of the precision of the measurement is obtained.

The four-transducer array provides six time-difference measurements since pairs of transducers can be selected in six ways. Triangulation equations for the array are:

$$
\begin{aligned}
& \theta_{2}-\theta_{1}:=a=\frac{v}{R}\left(t_{2}-t_{1}\right), . \\
& \theta_{3}-\theta_{1}=b=\frac{v}{R}\left(t_{3}-t_{1}\right), \\
& \theta_{3}-\theta_{2}=c=\frac{v}{R}\left(t_{3}-t_{2}\right), \\
& \theta_{3}-\theta_{4}=d=\frac{v}{R}\left(t_{3}-t_{4}\right) ; \\
& \theta_{1}-\theta_{4}=e=\frac{v}{R}\left(t_{1}-t_{4}\right) ; \text { and } \\
& \theta_{2}-\theta_{4}=f=\frac{v}{R}\left(t_{2}-t_{4}\right) .
\end{aligned}
$$

These equations are a set of linear equations for determining angles $\left(\theta_{1}, \theta_{2}, \theta_{3}\right.$; and $\left.\theta_{4}\right)$ in terms of time differences $(a, b, c, d, e$, and $f)$. This set of equations is of Rank 3 and hence only three are independent. The quantities $(a, b, c, d$, $e$, and f) would thus ideally satisfy the relations:

$$
\begin{aligned}
& c=b-a, \\
& b=d-e, \text { and } \\
& f=a+e,
\end{aligned}
$$


which can be rearranged as:

$$
\begin{gathered}
a=f-e, \\
b=d-e, \text { and } \\
c=d-f .
\end{gathered}
$$

The angles $\left(\theta_{1}, \theta_{2}, \theta_{3}\right.$, and $\left.\theta_{4}\right)$ are not all independent, since fixing values of any three will define a point on the sphere. For the transducer configuration shown in Figure 1, Angles $\theta_{1}$ and $\theta_{4}$ are related by:

$$
\theta_{4}=\pi-\theta_{1},
$$

which allows the (11) equations to be written as:

$$
\begin{gathered}
\theta_{2}-\theta_{1}=a, \\
\theta_{3}-\theta_{1}=b, \\
\theta_{3}-\theta_{2}=c, \\
\theta_{3}+\theta_{1}=\pi+d, \\
\theta_{1}=(\pi+e) / 2, \text { and } \\
\theta_{2}+\theta_{1}=\pi+f .
\end{gathered}
$$

In principle, any three of the set of (15) equations could be used to solve for $\theta_{1}, \theta_{2}$, and $\theta_{3}$. Since there are 20 ways that these equations can be selected, there should be an equal number of equivalent solutions. Of the 20 sets of three equations, four sets are of Rank 2 and cannot be solved explicitly for $\theta_{1}, \theta_{2}$, and $\theta_{3}$. These four sets of three equations involve time-difference measurements from only three of the transducers; and, hence, a unique solution is not obtained. In each case, the use of a fourth equation would give a unique solution. The remaining 16 sets of three equations are of Rank 3 and provide a unique solution. The 20 combinations of equations and their corresponding solutions are:

$$
\begin{gathered}
(a, b, c) \text { rank }=2- \\
\theta_{1}=\theta_{3}-b, \text { and } \\
\theta_{2}=\theta_{3}-c=\theta_{3}+a-b ;
\end{gathered}
$$


$(a, b, d)$ rank $=3-$

$$
\begin{gathered}
\theta_{1}=\frac{\pi+d-b}{2}, \\
\theta_{2}=\frac{2 a+d-b+\pi}{2}, \text { and } \\
\vartheta_{3}=\frac{\pi+d+b}{2} ;
\end{gathered}
$$

$(a, b, e)$ rank $=3-$

$$
\begin{gathered}
\theta_{1}=\frac{\pi+e}{2}, \\
\theta_{2}=\frac{2 a+e+\pi}{2} \text {, and } \\
\theta_{3}=\frac{2 b+\pi+e}{2} ;
\end{gathered}
$$

$(a, b, f)$ rank $=3-$

$$
\begin{gathered}
\theta_{1}=\frac{f-a+\pi}{2}, \\
\theta_{2}=\frac{a+f+\pi}{2}, \text { and } \\
\theta_{3}=\frac{2 b-a+\pi+f}{2} ;
\end{gathered}
$$

(a, c, d) rank $=3-$

$$
\begin{gathered}
\theta_{1}=\frac{d-a-c+\pi}{2}, \\
\theta_{2}=\frac{a-c+d+\pi}{2}, \text { and } \\
\theta_{3}=\frac{a+c+d+\pi}{2}
\end{gathered}
$$


28

$$
\begin{gathered}
(a, c, e) \text { rank }=3- \\
\vartheta_{1}=\frac{e+\pi}{2}, \\
9_{2}=\frac{2 a+e+\pi}{2}, \text { and } \\
9_{3}=\frac{2 a+2 c+e+\pi}{2} ;
\end{gathered}
$$

$$
\begin{aligned}
(a, c, f) \text { rank } & =3- \\
\theta_{1} & =\frac{f-a+\pi}{2}, \\
\theta_{2} & =\frac{a+f+\pi}{2}, \text { and } \\
\vartheta_{3} & =\frac{2 c+a+f+\pi}{2} ;
\end{aligned}
$$

$$
\begin{gathered}
(a, d, e) \text { rank }=3- \\
\theta_{1}=\frac{\pi+e}{2}, \\
\vartheta_{2}=\frac{2 a+e+\pi}{2}, \text { and } \\
\theta_{3}=\frac{2 d-e+\pi}{2} ;
\end{gathered}
$$

$$
\begin{aligned}
(a, d, f) \text { rank } & =3- \\
\theta_{1} & =\frac{f-a+\pi}{2}, \\
\theta_{2} & =\frac{a+f+\pi}{2}, \text { and } \\
\theta_{3} & =\frac{a+2 d-f+\pi}{2} ;
\end{aligned}
$$


$(a, e, f)$ rank $=2$

$$
\begin{gathered}
\theta_{1}=\frac{\pi+e}{2}, \\
\theta_{2}=\frac{2 a+\pi+e}{2}, \text { and } \\
\theta_{3}=\text { indeterminate; }
\end{gathered}
$$

(b, c, d) rank = $3-$

$$
\begin{gathered}
\theta_{1}=\frac{d-b+\pi}{2}, \\
\theta_{2}=\frac{b+d-2 c+\pi}{2}, \text { and } \\
\theta_{3}=\frac{b+d+\pi}{2}
\end{gathered}
$$

(b, c, e) rank = $3-$

$$
\begin{gathered}
\theta_{1}=\frac{\pi+e}{2}, \\
\theta_{2}=\frac{2 b-2 c+e+\pi}{2}, \text { and } \\
\theta_{3}=\frac{2 b+e+\pi}{2}
\end{gathered}
$$

(b, c, f) rank $=3-$

$$
\begin{gathered}
\theta_{1}=\frac{c-b+f+\pi}{2}, \\
\theta_{2}=\frac{b-c+f+\pi}{2}, \text { and } \\
\theta_{3}=\frac{c+b+f+\pi}{2}
\end{gathered}
$$


$(b, d, e)$ rank $=2-$

$$
\begin{gathered}
\theta_{1}=\frac{\pi+e}{2}, \\
\theta_{2}=\text { indeterminate, and } \\
\theta_{3}=\frac{2 b+\pi+e}{2} ;
\end{gathered}
$$

$(b, d, f)$ rank $=3-$

$$
\begin{gathered}
\theta_{1}=\frac{d-b+\pi}{2}, \\
\theta_{2}=\frac{b+2 f-d+\pi}{2}, \text { and } \\
\theta_{3}=\frac{b+d+\pi}{2} ;
\end{gathered}
$$

$(b, e, f)$ rank $=3-$

$$
\begin{gathered}
\theta_{1}=\frac{\pi+e}{2} ; \\
\theta_{2}=\frac{2 f-e+\pi}{2}, \text { and } \\
\theta_{3}=\frac{2 b+\pi+e}{2} ;
\end{gathered}
$$

(c, d, e) rank $=3-$

$$
\begin{gathered}
\theta_{1}=\frac{\pi+e}{2}, \\
\theta_{2}=\frac{2 d-2 c-e+\pi}{2}, \text { and } \\
\theta_{3}=\frac{2 d-e+\pi}{2}
\end{gathered}
$$




$$
\begin{aligned}
\text { (c, d, f) rank } & =2- \\
\theta_{1}= & -\theta_{3}+\pi+d, \text { and } \\
\theta_{2} & =\theta_{3}-c ; \\
(c, e, f) \text { rank } & =3- \\
& \theta_{1}=\frac{\pi+e}{2}, \\
\theta_{2} & =\frac{2 f-e+\pi}{2}, \text { and } \\
\theta_{3}= & \frac{2 c+2 f-e+\pi}{2} ; \\
\text { (d, e, f) rank } & =3- \\
\theta_{1} & =\frac{(\pi+e)}{2}, \\
\theta_{2} & =\frac{2 f-e+\pi}{2}, \text { and } \\
\theta_{3} & =\frac{2 d-e+\pi}{2} .
\end{aligned}
$$

Equating corresponding values of $\theta_{1}, \theta_{2}$, or $\theta_{3}$ in these different solutions will always produce one of the identities given in the (12) and (13) equations. The angles $\left(\theta_{1}, \theta_{2}\right.$, and $\left.\theta_{3}\right)$ are easily converted to spherical coordinate angles $(\theta, \varphi)$ when the solution is of Rank 3. All of these solutions have the form:

$$
\begin{gathered}
\theta_{1}=c_{1}=\cos ^{-1}(\sin \varphi \cdot \cos \theta), \\
\theta_{2}=c_{2}=\cos ^{-1}(\sin \varphi \cdot \sin \theta), \text { and } \\
\theta_{3}=c_{3}=\varphi,
\end{gathered}
$$

where $\left(c_{1}, c_{2}\right.$, and $\left.c_{3}\right)$ are the appropriate functions of $(a, b, c, d, e$, and $f)$. Solving the (16) equations for $\theta$ and $\varphi$ gives:

$$
\sin \theta=\frac{\cos c_{2}}{\sin c_{3}}
$$




$$
\begin{gathered}
\cos \theta=\frac{\cos c_{1}}{\sin c_{3}}, \text { and } \\
\varphi=c_{3} .
\end{gathered}
$$

Computer solution of the (17) equations for all cases having Rank 3 would provide sixteen flaw sites, and the diameter of this cluster of solutions would indicate the precision of the measurement. If only three transducers are used, a configuration can be selected which corresponds to one of the four Rank 2 solutions. In this case, the equations would likely indicate two valid locations for the flaw. 